\title{
Statistical Anisotropy in Imperfect Electromagnetic Reverberation
}

\author{
Luk R. Arnaut, Ramiro Serra, and Philip D. West
}

\begin{abstract}
A probabilistic characterization is performed for a class of field anisotropy coefficients for random electromagnetic fields. Ideal reverberation (isotropy), anisotropic stirring (polarization bias), and incomplete stirring or line-of-sight coupling (direct illumination) are investigated individually. For small degrees of planar anisotropy or bias, trapezoidal distributions are obtained, representing first-order departures from ideal rectangular distributions. Distributions for two definitions of total field anisotropies are found to be less sensitive than those for the individual planar anisotropies. The method and results are useful for calibration and validation of reverberation chambers when field anisotropy requires a separate assessment. Theoretical results are compared to measured data in two chambers.
\end{abstract}

Keywords: reverberation chamber, statistical anisotropy, validation of test sites.

\section{INTRODUCTION}

An ideal mode-tuned or mode-stirred reverberation chamber (MT/MSRC) exhibits a statistically homogeneous, isotropic, and randomly polarized field, by definition. A realistic chamber has nonzero levels of field inhomogeneity, anisotropy, or polarization bias that characterize its stirring performance. Quantifying these imperfections in an empty chamber is important for EMC testing, particularly for uncertainty quantification. To this end, the standard normative method for validating MT/MSRCs in [1, Appendix B] uses a combination of (local) field isotropy and (nonlocal) homogeneity measurements. These are taken along three orthogonal directions at eight locations spanning the working volume. All measured data are then combined to compute a single measure of field nonuniformity.

In some applications, however, the required statistical uniformity may be limited to field randomization at just a single location - more generally, in an electrically small region i.e., statistical field isotropy (spherical angular uniformity of polarization) only. This is separate from homogeneity (positional uniformity) between different locations, for an arbitrary direction. Thus, when only statistical isotropy is of interest, the normative IEC metric which intertwines field anisotropy with inhomogeneity is unsuitable and it may be unnecessarily stringent. Even when both isotropy and homogeneity are of interest, the summation of field components for different directions with those at different locations tacitly assumes ergodicity to hold, in order to yield meaningful statistics such as this IEC metric. The assumption of ergodicity only holds asymptotically, in the high-frequency limit, and is often inapplicable by a margin near the lowest usable frequency (LUF).
In other applications, pronounced levels of field anisotropy may be sought intentionally. For example, in immunity testing of printed circuit boards that are idealized as a 2-D equipment under test (EUT), a statistically planar test E-field with minimized aspect ratio for the perpendicular component to the tangential component is typically preferential because of its stronger excitations. In emissions, the characteristics of spatially concentrated EM sources (including apertures, vias, etc.) may differ significantly from those by spatially extended inhomogeneous sources because of interference. In yet other scenarios, statistical field anisotropy may be inherent, e.g., near a PEC boundary for floor standing EUTs, where confidence limits at different heights are of interest. Thus, the full probabilistic characterization of a random field with aspect ratios $\sigma_{E_{i}}(\underline{r}): \sigma_{E_{j}}(\underline{r}): \sigma_{E_{k}}(\underline{r})$ at an arbitrary $\underline{r}$ is of interest, where $\sigma_{E_{i}}$ is the standard deviation of component $E_{i}$.

Ideal (perfect) reverberation exhibits a circular Gaussian random field. Imperfections [2] can be assessed by comparing theoretical and empirical probability density functions (pdfs) or cumulative distribution functions (cdfs) for the field magnitude or energy using hypothesis testing, e.g., using Pearson $\left(\chi^{2}\right)$ [3], Kolmogorov-Smirnov [4], Cramér-von Mises [5], or Anderson-Darling [6] tests. It is desirable to have alternative higher-level metrics that assess anisotropy and inhomogeneity in a direct manner and enable physical interpretation, in which statistical distributions of the field components are useful but auxiliary ingredients. Analysis of variance (ANOVA) methods to test the three-way null hypothesis of isotropy, $H_{0}: \sigma_{E_{i}}^{2}(\underline{r})=$ $\sigma_{E_{j}}^{2}(\underline{r})=\sigma_{E_{k}}^{2}(\underline{r})$, are limited because these require Gaussianity (together with homoskedasticity and independence).

To provide a less restrictive and more refined ${ }^{1}$ quantification of field anisotropy, a class of planar $\left(A_{i j}\right)$ and total $(A)$ field anisotropy coefficients was introduced in [7] and [8, Appendix]. These coefficients were shown to provide practical and consistent metrics of local reverberation performance [1, Annex J]. In this paper, the focus is on their probabilistic characterization, including scenarios of understirring and residual line-of-sight coupling (direct illumination, unstirred field contributions). Although statistical field isotropy, homogeneity, and degree of polarization typically appear to be correlated [9, Fig. 7.1], this does not prevent their individual statistical characterization, at least on a marginal-probabilistic basis. Furthermore, an alternative definition for the total vector field anisotropy, $A^{\prime}$, is introduced that renormalizes $A$ in case of unequal planar energies for pairs of spatial orientations. Theoretical results are compared with Monte Carlo simulations

${ }^{1}$ in the sense of pairwise comparison of field components 
and measurements in two different chambers

Throughout this paper, an $\exp (\mathrm{j} \omega t)$ time dependence is assumed and suppressed. The usual convention of denoting random variables with upper case and their corresponding realized values with lower case symbols is adopted. For brevity, the adjective 'statistical' for field (an)isotropy is omitted. Secs. III-B and IV summarize an earlier theoretical analysis [7].

\section{DEFINITION OF FiELD ANisotropy}

Real or complex random isotropic fields (also known as spherical, radial, or rotationally invariant fields) preserve their pdf under any orthogonal or unitary transformation, respectively [10]. For stirred cavities evaluated at an arbitrary interior location $\underline{r}$, a simplified and pragmatic definition for statistical isotropy based on the intensity of the electric field $E$ is

$$
\left\langle\left|E_{x}(\underline{r})\right|^{2}\right\rangle=\left\langle\left|E_{y}(\underline{r})\right|^{2}\right\rangle=\left\langle\left|E_{z}(\underline{r})\right|^{2}\right\rangle,
$$

where the intensities of the Cartesian field components, $\left|E_{i}\right|^{2}$ $(i=x, y, z)$ - which can be replaced by energies or powers - are measured at steady-state boundary configurations $t_{j}$ in a discrete mode tuning process. The ensemble averages $\langle\cdot\rangle$ in (1) can be approximated by sample averages obtained for a sufficiently large set of such configurations generated by this process. However, statistical independence of the $\left|E_{i}\left(t_{j}\right)\right|^{2}$ with respect to $t_{j}$ is not strictly required in our probabilistic (i.e., first-order) characterization. The medium filling the cavity is itself assumed to be isotropic, homogeneous, linear, and time-invariant, such that $D_{i}(\underline{r}, t)=\epsilon E_{i}(\underline{r}, t)$.

Field anisotropy can be quantified from three differences of intensities, associated with two of any three mutually orthogonal directions $\left(\underline{1}_{i}, \underline{1}_{j}, \underline{1}_{k}\right)$ and each pair normalized by their sum. Following the definitions in [8, eqs. (66) and (67)], with the foregoing assumptions on the filling medium, the coefficients defined by

$$
\begin{aligned}
A_{i j} & \triangleq \frac{\left|E_{i}\right|^{2}-\left|E_{j}\right|^{2}}{\left|E_{i}\right|^{2}+\left|E_{j}\right|^{2}}, \quad(i, j=x, y, z ; i \neq j) \\
A & \triangleq \sqrt{\frac{A_{x y}^{2}+A_{y z}^{2}+A_{z x}^{2}}{3}}
\end{aligned}
$$

represent planar and total anisotropy coefficients, respectively. The functional dependence of the $E_{i, j}$ on $t$ has been implied and suppressed. Note that the fields featuring in (2) and (3) are physical fields, unlike the amplitudes $\mathcal{E}_{n}$ associated with the $n$th component of an angular spectrum of plane waves with a nonphysical angle of arrival (incidence), whose isotropy has been studied in [11].

\section{Anisotropic Stir PERFORMANCE}

\section{A. Pdf of Fields and Energy}

Consider $E_{i} \equiv E_{i}^{\|}+\mathrm{j} E_{i}^{\perp}$, which is assumed to exhibit a centered circular Gauss normal pdf $\mathrm{N}\left(0, \sigma_{E_{i}}\right)$. This implies the following assumptions:

1) the in-phase and quadrature components $E_{i}^{\|}$and $E_{i}^{\perp}$ have equal standard deviations $\sigma_{E_{i}^{\|}}=\sigma_{E_{i}^{\perp}} \triangleq \sigma_{E_{i}}, \forall i$;

2) each Cartesian component $E_{i}$ is unbiased, i.e., $\mu_{E_{i}}=$ $\mu_{E_{i}^{\|}}=\mu_{E_{i}^{\perp}}=0, \forall i$, where $\mu_{E_{i}^{(\|)(\perp)}} \equiv\left\langle E_{i}^{(\|)(\perp)}\right\rangle$ represent ensemble mean (expected) values across all stir states;

3) $E_{i}^{\|}$and $E_{i}^{\perp}$, as well as $E_{i}$ and $E_{j}$, are statistically independent. $^{2}$

The first assumption is more general than what is usually considered, viz. ideal reverberation for which $\sigma_{E_{i}}$ is constant for all $i$ (identical distributions) [3], [12, Sec. 2.4]. The second assumption greatly simplifies the analysis and can be asserted provided direct illumination is absent [3], or it can be approximated by subtracting the sample mean in a preprocessing step. Nevertheless, this restriction will be lifted in Sec. IV-A2. For circular complex $E_{i}$, the variance of $\left|E_{i}\right|^{2}$ and its components $\mid E_{i}^{\|^{2}}$ and $\left|E_{i}^{\perp}\right|^{2}$ can be denoted, on account of the first assumption and for brevity, as

$$
\sigma_{i} \triangleq \sigma_{\left|E_{i}\right|^{2}}=\sigma_{E_{i}^{\|}}^{2}+\sigma_{E_{i}^{\perp}}^{2}=2 \sigma_{E_{i}}^{2} .
$$

\section{B. Planar Field Anisotropy $A_{i j}$}

The pdf $f_{A_{i j}}\left(a_{i j}\right)$ for $A_{i j}$ is derived in the Appendix, where it is shown to be

$f_{A_{i j}}\left(a_{i j}\right)=\frac{2 \sigma_{r}}{\left[\left(\sigma_{r}+1\right)+\left(\sigma_{r}-1\right) a_{i j}\right]^{2}}, \quad-1 \leq a_{i j} \leq 1$.

This pdf exhibits only one parameter, viz., the ratio of the variances of the Cartesian field intensities in (4), i.e., $\sigma_{r} \triangleq$ $\sigma_{j} / \sigma_{i}$. This parameter expresses the anisotropy of the stirring efficiency [5, Sec. 3.1.8] as an "aspect ratio" for the intensity of the random field in the plane. The corresponding cdf is

$$
F_{A_{i j}}\left(a_{i j}\right)=\frac{\left(1+a_{i j}\right) \sigma_{r}}{\left(\sigma_{r}+1\right)+\left(\sigma_{r}-1\right) a_{i j}}, \quad-1 \leq a_{i j} \leq 1
$$

The pdf and cdf are shown in Fig. 1 for selected values of $\sigma_{r}$. In general, the median $F_{A_{i j}}^{-1}(1 / 2)=\left(1-\sigma_{r}\right) /\left(1+\sigma_{r}\right)$

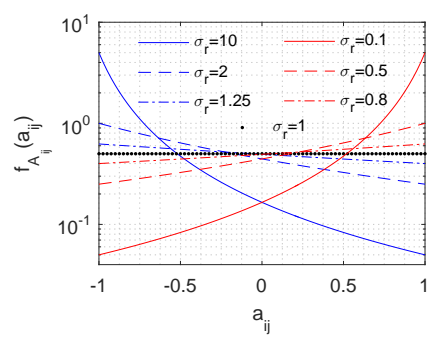

(a)

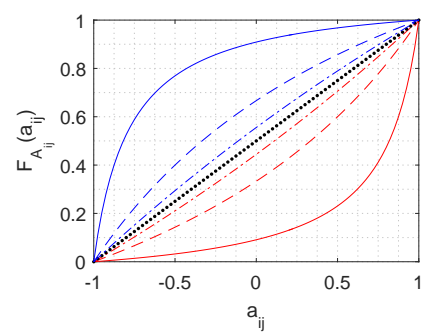

(b)
Fig. 1. (a) Pdfs $f_{A_{i j}}\left(a_{i j}\right)$ and (b) corresponding cdfs $F_{A_{i j}}\left(a_{i j}\right)$ (same line type and color code as for (a)) for unbiased fields $E_{i, j}$ at selected values of stirring anisotropy $\sigma_{r}=\sigma_{j} / \sigma_{i}$

does not coincide with $a_{i j}=0$ unless the stirring is isotropic, i.e., when $\sigma_{i}=\sigma_{j}$. The symmetry of the pdf depends on $\sigma_{r}$, because $\int_{0}^{1} f_{A_{i j}}\left(a_{i j}\right) \mathrm{d} a_{i j}=\sigma_{r} /\left(1+\sigma_{r}\right)$ for half of the range of $a_{i j}$ is in general different from $1 / 2$. For $\sigma_{i}=\sigma_{j} \triangleq \sigma$, the distribution is uniform (rectangular), i.e.,

$f_{A_{i j}}\left(a_{i j}\right)=\frac{1}{2}, F_{A_{i j}}\left(a_{i j}\right)=\frac{1+a_{i j}}{2}, \quad-1 \leq a_{i j} \leq 1$.

\footnotetext{
${ }^{2}$ Statistical dependence can be included by introducing a nonzero covariance between two or more field components.
} 
Thus, in ideal reverberation the planar field anisotropy is not a constant but has constant probability density across its range $[-1,+1]$. In particular, it is then independent of $\sigma_{\left|E_{i}\right|^{2}}$ and $\sigma_{\left|E_{j}\right|^{2}}$ (mean square fluctuations) for the respective spatial field components that span the plane of observation oij.

The mean and standard deviation of $A_{i j}$ follow as

$$
\begin{aligned}
\mu_{A_{i j}} & =\frac{1+2 \sigma_{r} \ln \sigma_{r}-\sigma_{r}^{2}}{\left(\sigma_{r}-1\right)^{2}}, \\
\sigma_{A_{i j}} & =\frac{2 \sqrt{\sigma_{r}-\left(2+\ln ^{2} \sigma_{r}\right) \sigma_{r}^{2}+\sigma_{r}^{3}}}{\left(\sigma_{r}-1\right)^{2}} .
\end{aligned}
$$

If $\sigma_{r}=1$ then $\mu_{A_{i j}}=0$ and $\sigma_{A_{i j}}=1 / 3$. The dependence of $\mu_{A_{i j}}$ and $\sigma_{A_{i j}}$ on $\sigma_{r}$ together with their ratio (coefficient of variation) $\nu_{A_{i j}} \triangleq \sigma_{A_{i j}} / \mu_{A_{i j}}$ is shown in Fig. 2 .

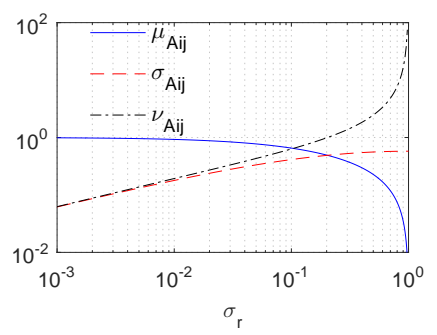

Fig. 2. Mean $\mu_{A_{i j}}$, standard deviation $\sigma_{A_{i j}}$ and coefficient of variation $\nu_{A_{i j}}$ as a function of stir anisotropy $\sigma_{r}$ for unbiased fields $E_{i, j}$.

If $\sigma_{i}$ and $\sigma_{j}$ are unknown, then these need estimation prior to determining $A_{i j}$. The ratio $\sigma_{r}$ can be estimated from measured values of $\left|E_{i}\right|^{2}$ and $\left|E_{j}\right|^{2}$, e.g., using moment method estimation or maximum likelihood estimation. It is recommended to calculate the estimate $\widehat{\sigma_{r}}$ from the data for the ratio $\left|E_{j}(t)\right|^{2} /\left|E_{i}(t)\right|^{2}$ per stir state, rather than as the ratio of the estimates $\widehat{\sigma}_{j} / \widehat{\sigma}_{i}$ from the individual data $\left\{\left|E_{j}\right|^{2}\right\}_{t}$ and $\left\{\left|E_{i}\right|^{2}\right\}_{t}$, because the former estimate reduces the effect of field or power calibration errors on $\widehat{\sigma_{r}}$, particularly when $\left|E_{i}\right|^{2} \ll\left|E_{j}\right|^{2}$ or $\left|E_{i}\right|^{2},\left|E_{j}\right|^{2} \simeq 0$.

Fig. 3 compares the theoretical and empirical cdfs. Measurements were taken using a three-axis E-field probe inside a mode-tuned stadium reverberation chamber at NPL (internal volume $V \simeq 0.2 \mathrm{~m}^{3}$ with inner average diameter $\simeq 0.7 \mathrm{~m}$ ), using rotational wall stirring generated by diffusing spherical caps as corrugations [5]. Measurements were taken in the equatorial plane at a radial distance $r \equiv z$ from the axis of rotation $o x$, equivalent to a distance $d=0.35-r$ to the nearest wall measured along $o z$. Data of $\left|E_{x}\right|,\left|E_{y}\right|$ and $\left|E_{z}\right|$ were measured across one full rotation of the wall, at $8.2 \mathrm{GHz}$ for 1200 stir states (green curves; characteristic electrical size $k V^{1 / 3}=97.0$ ) and at $2.5 \mathrm{GHz}$ for 275 stir states (blue and red curves; $k V^{1 / 3}=29.6$ ). Estimates $\widehat{\sigma_{x, y, z}}=s_{x, y, z}$ obtained from the data serve as parameter values for the theoretical cdfs. It is seen that $F_{A_{i j}}\left(a_{i j}\right)$ and $F_{A}(a)$ approach the cdfs for ideal isotropy more closely at the higher frequency and deeper inside the cavity, as expected, whereas large anisotropies occur when the wall is approached, on account of the EM boundary conditions for $E$. Although measurements and tests are typically chosen to be performed "far" from any wall so that $d=9 \mathrm{~mm}$ would not be a location for normal $\mathrm{RC}$ operation, some scenarios enforce such small distances, e.g., an electronic component near a shielding enclosure, where proximity effects would be observed. The consistency of the results for $F_{A_{i j}}\left(a_{i j}\right)$ when approaching a wall has been shown in more detail in [13].

Fig. 3 shows how the theoretical distributions can be used in practice: for a specified desired level $\sigma_{r}$, the theoretical cdf (6) is computed and compared against the empirical cdf, either through a goodness-of-fit hypothesis testing or by requiring a certain fraction of the data to fall within a band $F_{A_{i j}}\left[a_{i j} \pm\right.$ $\delta)] \pm \varepsilon$ around the theoretical ${ }^{3} F_{A_{i j}}\left(a_{i j}\right)$, for chosen tolerances $\delta$ and $\varepsilon$.
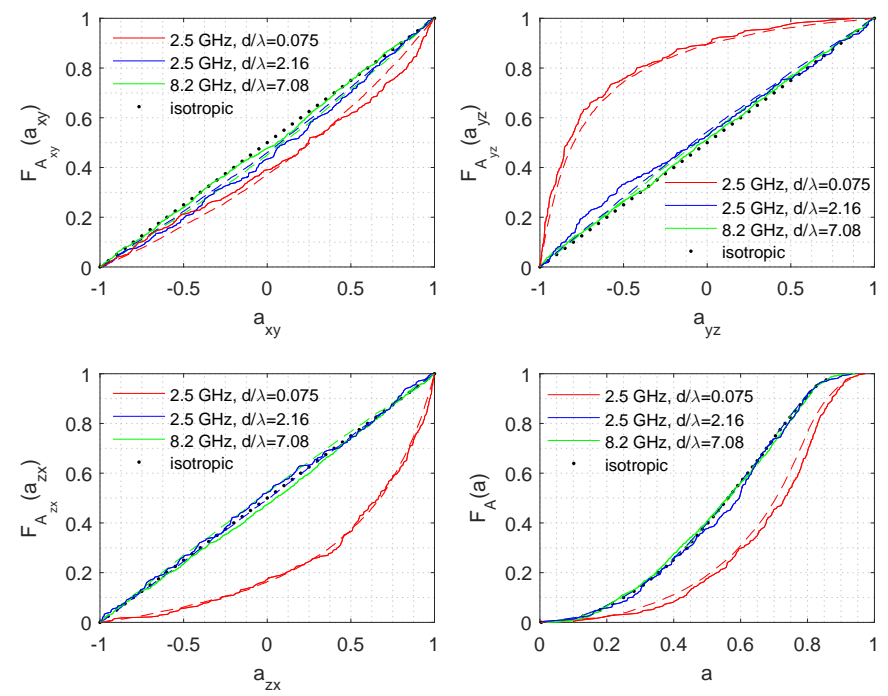

Fig. 3. Comparison of theoretical (dashed) vs. empirical (solid) ensemble cdfs of $A_{i j}(i, j=x, y, z)$ and $A$, for data measured at distance $d$ to nearest chamber wall (green and blue: $d=259 \mathrm{~mm}$; red: $d=9 \mathrm{~mm}$ ). Ensemble cdfs of $A_{i j}$ and $A$ for ideal isotropic fields (black dots) are shown for reference.

\section{Total Field Anisotropy A}

1) Unweighted Planar Energies: The total field anisotropy $A$ in (3) can be rewritten in terms of $X_{i, j, k} \triangleq\left|E_{i, j, k}\right|^{2}$ as

$A=\sqrt{\frac{1}{3}\left[\left(\frac{X_{i}-X_{j}}{X_{i}+X_{j}}\right)^{2}+\left(\frac{X_{j}-X_{k}}{X_{j}+X_{k}}\right)^{2}+\left(\frac{X_{k}-X_{i}}{X_{k}+X_{i}}\right)^{2}\right]}$.

Since $X_{i}, X_{j}$ and $X_{k}$ are assumed to be independent, i.e., $f_{X_{i}, X_{j}, X_{k}}\left(x_{i}, x_{j}, x_{k}\right)=f_{X_{i}}\left(x_{i}\right) f_{X_{j}}\left(x_{j}\right) f_{X_{k}}\left(x_{k}\right)$, the cdf of $A$ is

$$
F_{A}(a)=\iiint_{\mathcal{R}(a)} f_{X_{i}}\left(x_{i}\right) f_{X_{j}}\left(x_{j}\right) f_{X_{k}}\left(x_{k}\right) \mathrm{d} x_{i} \mathrm{~d} x_{j} \mathrm{~d} x_{k},
$$

valid for $0 \leq a \leq 1$ where $f_{X_{i, j, k}}\left(x_{i, j, k}\right) \sim \chi_{2}^{2}\left(\sigma_{i, j, k}\right)$. The region of integration $\mathcal{R}(a)$ is the volume defined by

$\mathcal{R}(a)=\left\{\left(x_{i}, x_{j}, x_{k}\right) \in\left(\mathbb{R}^{3}\right)^{+}: 0 \leq \psi\left(x_{i}, x_{j}, x_{k}\right) \leq a\right\}$,

${ }^{3}$ assuming a sufficiently large set of statistically independent data, so that ensemble distributions are applicable; for sampling cdfs in case of relatively small sample sets, cf. [13, Sec. IV]. 


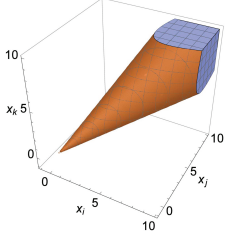

(a)

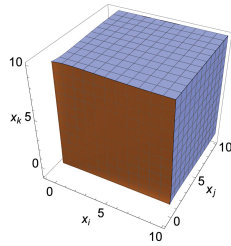

(c)

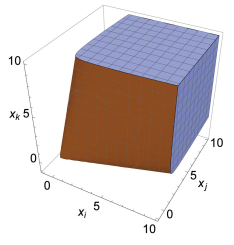

(b)

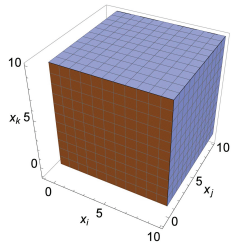

(d)
Fig. 4. Three-dimensional plots of the region of integration $\mathcal{R}(a)$ for selected values of $a$ : (a) $a=0.2$, (b) $a=0.6$, (c) $a=a_{c} \simeq 0.816$, and (d) $a=1$.

located in the first (i.e., triple positive) octant $\left(\mathbb{R}^{3}\right)^{+}$, with

$\psi\left(x_{i}, x_{j}, x_{k}\right) \triangleq \sqrt{\frac{1}{3}\left[\left(\frac{x_{i}-x_{j}}{x_{j}+x_{j}}\right)^{2}+\left(\frac{x_{j}-x_{k}}{x_{j}+x_{k}}\right)^{2}+\left(\frac{x_{k}-x_{i}}{x_{k}+x_{i}}\right)^{2}\right]}$

Geometrically, $\mathcal{R}(a)$ is a non-circular solid cone in $\left(\mathbb{R}^{3}\right)^{+}$with its apex located at the origin and apex angle increasing with $a$. Fig. 4 shows $\mathcal{R}(a)$ for selected values of $a$. For $a=0$, the cone collapses to the line $x=y=z$, resulting in $F_{A}(0)=0$. yielding the pdf $f_{A}(a) \equiv \mathrm{d} F_{A}(a) / \mathrm{d} a$ as$$
f_{A}(a)=\iint_{\mathcal{S}_{-}(a)} \frac{f_{X_{i}, X_{j}, X_{k}}\left(x_{i}, x_{j}, x_{k}\right)}{\left\|\nabla \psi\left(x_{i}, x_{j}, x_{k}\right)\right\|} \mathrm{d} x_{i} \mathrm{~d} x_{j} \mathrm{~d} x_{k},
$$

For $a=a_{c} \triangleq \sqrt{2 / 3} \simeq 0.816$, the cone is on the verge of intersecting the coordinate planes, producing a discontinuity in the slope of $f_{A}(a)$. For $a=1, \mathcal{R}(a)$ coincides with $\left(\mathbb{R}^{3}\right)^{+}$ and yields $F_{A}(1)=1$. The numerical evaluation of (11) yields $F_{A}(a)$ for all $a$, as shown in Fig. 5 for selected values of $\sigma_{i, j, k}$. The main effect of reverberation imperfections on $A$ (i.e., diverging $\left.\sigma_{i, j, k}\right)$ is an increase of $f_{A}(a)$ for $\left.a\right\rangle\langle A\rangle \simeq$ 0.541 . These deviations also increase the median $F_{A}^{-1}(1 / 2)$. The minimum median occurs for ideal isotropic reverberation $\left(\sigma_{i}=\sigma_{j}=\sigma_{k}\right)$.

The pdf $f_{A}(a) \equiv \mathrm{d} F_{A}(a) / \mathrm{d} a$ can be found by computing $F_{A}(a+\mathrm{d} a)-F_{A}(a)$ to first order in $\mathrm{d} a$, which yields

$$
\begin{aligned}
& F_{A}(a+\mathrm{d} a)-F_{A}(a)= \\
& \quad \iiint_{\mathcal{S}(a)} f_{X_{i}, X_{j}, X_{k}}\left(x_{i}, x_{j}, x_{k}\right) \mathrm{d} x_{i} \mathrm{~d} x_{j} \mathrm{~d} x_{k},
\end{aligned}
$$

where $f_{X_{i}, X_{j}, X_{k}}\left(x_{i}, x_{j}, x_{k}\right)$ is again the joint pdf of $X_{i}, X_{j}$ and $X_{k}$, whilst the domain of integration is now

$$
\mathcal{S}(a) \triangleq\left\{x_{i, j, k} \in\left(\mathbb{R}^{3}\right)^{+}: a \leq \psi\left(x_{i}, x_{j}, x_{k}\right) \leq a+\mathrm{d} a\right\}
$$

which represents a shell, i.e., a hollow cone with inner and outer conical surfaces $\mathcal{S}_{-}(a): \psi\left(x_{i}, x_{j}, x_{k}\right)=a$ and $\mathcal{S}_{+}(a): \psi\left(x_{i}, x_{j}, x_{k}\right)=a+\mathrm{d} a$, with infinitesimal thickness $\epsilon=\mathrm{d} a /\left\|\nabla \psi\left(x_{i}, x_{j}, x_{k}\right)\right\|$. Eq. (14) can thus be rewritten as

$$
\begin{aligned}
& F_{A}(a+\mathrm{d} a)-F_{A}(a)= \\
& \quad \iiint_{\mathcal{S}_{-}(a)} \epsilon f_{X_{i}, X_{j}, X_{k}}\left(x_{i}, x_{j}, x_{k}\right) \mathrm{d} x_{i} \mathrm{~d} x_{j} \mathrm{~d} x_{k},
\end{aligned}
$$

in which the integration is now performed across $\mathcal{S}_{-}(a)$. Geometrically, $\mathcal{S}_{-}(a)$ is the outer surface of the volume $\mathcal{R}(a)$, shown in orange color in Fig. 4. The final expression of the integral reads

$$
f_{A}(a)=\iiint_{\mathcal{S}_{-}(a)} \frac{\frac{s q r t 3}{\sigma_{i} \sigma_{j} \sigma_{k}} \sqrt{\left(\frac{x_{i}-x_{j}}{x_{i}+x_{j}}\right)^{2}+\left(\frac{x_{j}-x_{k}}{x_{j}+x_{k}}\right)^{2}+\left(\frac{x_{k}-x_{i}}{x_{k}+x_{i}}\right)^{2}} \exp \left[-\left(\frac{x_{i}}{\sigma_{i}}+\frac{x_{j}}{\sigma_{j}}+\frac{x_{k}}{\sigma_{k}}\right)\right]}{2 \sqrt{\left(\frac{x_{j}\left(x_{i}-x_{j}\right)}{\left(x_{i}+x_{j}\right)^{3}}+\frac{x_{k}\left(x_{i}-x_{k}\right)}{\left(x_{i}+x_{k}\right)^{3}}\right)^{2}+\left(\frac{x_{i}\left(x_{j}-x_{i}\right)}{\left(x_{j}+x_{i}\right)^{3}}+\frac{x_{k}\left(x_{j}-x_{k}\right)}{\left(x_{j}+x_{k}\right)^{3}}\right)^{2}+\left(\frac{x_{j}\left(x_{k}-x_{j}\right)}{\left(x_{k}+x_{j}\right)^{3}}+\frac{x_{i}\left(x_{k}-x_{i}\right)}{\left(x_{k}+x_{i}\right)^{3}}\right)^{2}}} \mathrm{~d} x_{i} \mathrm{~d} x_{j} \mathrm{~d} x_{k} .
$$

Eq. (18) can be directly evaluated by numerical quadrature when $a \lesssim 0.6$. For $0.6<a \leq 1$, numerical convergence becomes problematic. Therefore, $f_{A}(a)$ is most efficiently calculated piecewise: in $a \in[0,0.6]$, numerical integration of (18) is performed, whereas in $a \in(0.6,1]$ numerical differentiation is applied to the numerically evaluated cdf (11). To avoid numerical instability, the finite-difference approximation to the differentiation can be implemented using a linear filter that yields the derivative for a cubic spline interpolation model of the cdf as input to the filter. To suppress numerical noise, the cdf data were regularized by a Gaussian kernel with a standard deviation of unity.

In Fig. 6, the numerically computed $f_{A}(a)$ obtained from (17) is compared to a Monte Carlo (MC) simulation and to measured data. For the simulation, three sets of $\chi_{2}$-distributed samples were generated by computer, thus emulating independent realizations of $\left|E_{i}\right|^{2}$ for perfectly reverberant fields. A relatively high number of samples was chosen $\left(N=10^{8}\right)$, in order to obtain a reference pdf for further comparisons. The frequency polygon of $A$ was calculated for these synthetic data 


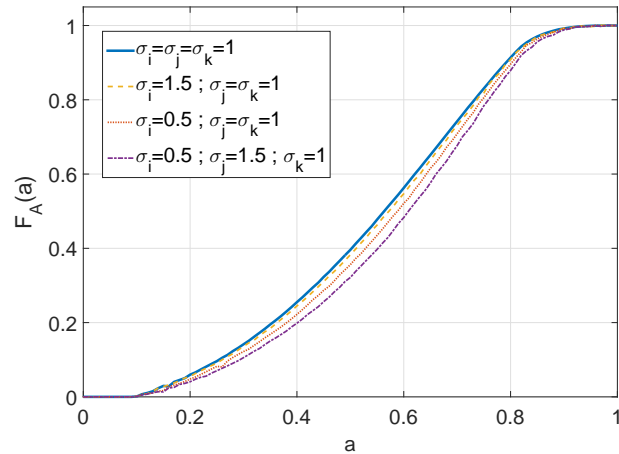

Fig. 5. Cdf of total field anisotropy $A$ for selected values of the triplets $\sigma_{i, j, k}$, representing deviations from ideal reverberation.

and is plotted in Fig. 6. Measured data for $E_{i}$ were obtained in the chamber at TU Eindhoven using a three-axial E-field sensor at nine different locations inside the working volume. The chamber was excited by a $\mathrm{CW}$ signal at $1 \mathrm{GHz}$, which is well above the lowest usable frequency for this chamber of about $200 \mathrm{MHz}$. The field was sampled at 200 equally spaced positions of the stirrer. The total anisotropy $A$ was calculated and the frequency polygons of the nine curves are plotted in Fig. 6. Good correspondence is obtained between the numerically computed $f_{A}(a)$ based on (17) and the MC simulation. All nine frequency polygons for the measured data fluctuate around (17) accordingly. In particular, the empirical $f_{A}(a)$ shows increased fluctuation in the region $0.6 \lesssim a \leq 0.8$, as an artefact of the finite-differencing approximation. Increasing the working precision was found to lead to smoother curves. Remaining fluctuations of the empirical $f_{A}(a)$ outside this interval are due to the finite number of stirrer positions. In Fig.

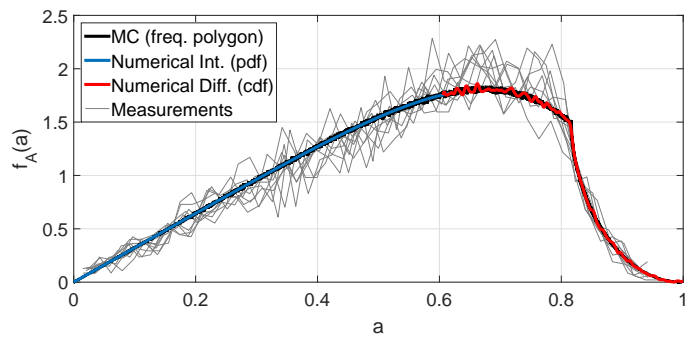

Fig. 6. Pdf $f_{A}(a)$ computed from (17) and compared against MC simulations and empirical distribution based on measured data.

6 , an abrupt change in the slope of $f_{A}(a)$ at the corner point $a_{c}$ is evident. This value $a_{c}$ is attained when one variate vanishes while the other two are equal, e.g., at $x_{i}=0, x_{j}=x_{k}$. This condition yields $\psi=a_{c}$ and introduces a discontinuity in $\mathcal{R}(a)$ as a result of the intersection of the coordinate planes by $\mathcal{S}_{-}(a)$, reflected in a corresponding discontinuity of the slope of the theoretical $f_{A}(a)$.

2) Weighted Planar Energies: The definition (3) tacitly assumes that the three planar anisotropies are combined with equal weight. This is justified provided the field energies associated with these planes are equal, i.e., $X_{i}+X_{j}=$ $X_{j}+X_{k}=X_{k}+X_{i}=2 X / 3$, where $X \triangleq X_{i}+X_{j}+X_{k}$. More

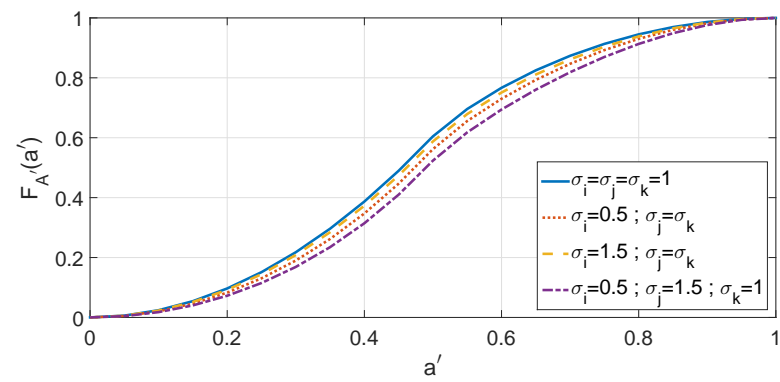

Fig. 7. Cdf of total field anisotropy $A^{\prime}$ for selected values of the triplets $\sigma_{i, j, k}$, representing deviations from ideal reverberation.

generally, when these planes carry unequal instantaneous energy, the corresponding $A_{i j}, A_{j k}$ and $A_{k i}$ should be weighted by respective fractions of energy $\left(X_{i}+X_{j}\right) /\left(X_{i}+X_{j}+X_{k}\right)$, etc., before recombination to an overall anisotropy coefficient. Thus, using (10) with the conversions

$\frac{X_{i}-X_{j}}{X_{i}+X_{j}} \rightarrow\left(\frac{3}{2} \frac{X_{i}+X_{j}}{X_{i}+X_{j}+X_{k}}\right) \frac{X_{i}-X_{j}}{X_{i}+X_{j}}=\frac{X_{i}-X_{j}}{2 X / 3}$,

etc., leads to the alternative definition

$$
A^{\prime} \triangleq \sqrt{\frac{1}{2 X^{2}}\left[\left(X_{i}-X_{j}\right)^{2}+\left(X_{j}-X_{k}\right)^{2}+\left(X_{k}-X_{i}\right)^{2}\right]},
$$

which has been renormalized in order to satisfy $A^{\prime}=1$ for the case of linear polarization.

Following a similar approach as in Sec. III-C1 for $A$, the cdf of $A^{\prime}$ is found as

$$
F_{A^{\prime}}\left(a^{\prime}\right)=\iiint_{\mathcal{R}^{\prime}\left(a^{\prime}\right)} f_{X_{i}}\left(x_{i}\right) f_{X_{j}}\left(x_{j}\right) f_{X_{k}}\left(x_{k}\right) \mathrm{d} x_{i} \mathrm{~d} x_{j} \mathrm{~d} x_{k}
$$

for $0 \leq a^{\prime} \leq 1$. The region of integration $\mathcal{R}^{\prime}\left(a^{\prime}\right)$ is now

$\mathcal{R}^{\prime}\left(a^{\prime}\right)=\left\{\left(x_{i}, x_{j}, x_{k}\right) \in\left(\mathbb{R}^{3}\right)^{+}: 0 \leq \psi^{\prime}\left(x_{i}, x_{j}, x_{k}\right) \leq a^{\prime}\right\}$,

with

$\psi^{\prime}\left(x_{i}, x_{j}, x_{k}\right) \triangleq \sqrt{\frac{\left(x_{i}-x_{j}\right)^{2}+\left(x_{j}-x_{k}\right)^{2}+\left(x_{k}-x_{i}\right)^{2}}{2\left(x_{i}+x_{j}+x_{k}\right)^{2}}}$.

Numerical evaluation of (21) for selected values of $\sigma_{i, j, k}$ yields the cdfs shown in Fig. 7. Comparing these $F_{A^{\prime}}\left(a^{\prime}\right)$ with $F_{A}(a)$ of Fig. 5 shows that even in the isotropic case $\left(\sigma_{i}=\sigma_{j}=\sigma_{k}\right)$, the cdfs are different because of the different normalization factor used to arrive at $F_{A^{\prime}}(1)=1$. The pdf of $A^{\prime}$ can be calculated by following the same steps as detailed in Sec. III-C1. This now results in

$$
f_{A^{\prime}}\left(a^{\prime}\right)=\iint_{\mathcal{S}_{-}^{\prime}\left(a^{\prime}\right)} \frac{f_{X_{i}, X_{j}, X_{k}}\left(x_{i}, x_{j}, x_{k}\right)}{\left\|\nabla \psi^{\prime}\left(x_{i}, x_{j}, x_{k}\right)\right\|} \mathrm{d} x_{i} \mathrm{~d} x_{j} \mathrm{~d} x_{k},
$$

where integration is along the surface $\mathcal{S}_{-}^{\prime}\left(a^{\prime}\right)$ given by

$$
\mathcal{S}_{-}^{\prime}\left(a^{\prime}\right) \triangleq\left\{x_{i, j, k} \in\left(\mathbb{R}^{3}\right)^{+}: \psi^{\prime}\left(x_{i}, x_{j}, x_{k}\right)=a^{\prime}\right\} .
$$


In Fig. 8, $f_{A^{\prime}}\left(a^{\prime}\right)$ is compared with the MC simulation result and with the same measured data used in Sec. III-C1. Again, an abrupt change in the slope of $f_{A^{\prime}}\left(a^{\prime}\right)$ is found for the points where $\mathcal{S}_{-}^{\prime}\left(a^{\prime}\right)$ intersects the coordinate planes, now resulting in a discontinuity in the slope occurring at a lower abscissa value, $a_{c}^{\prime}=0.5$. Table I lists summary statistics of $A$ and $A^{\prime}$

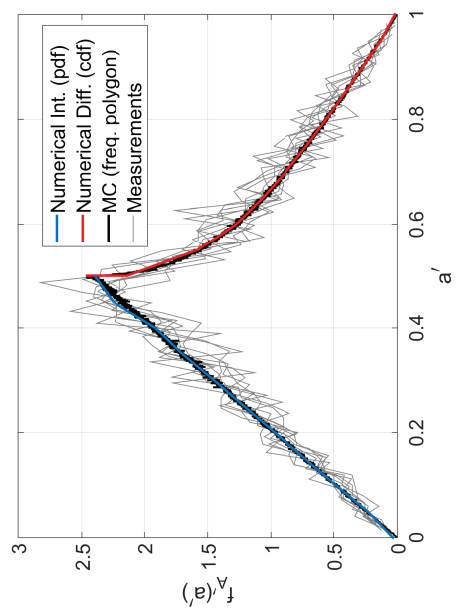

Fig. 8. $f_{A^{\prime}}\left(a^{\prime}\right)$ compared against MC simulations and measured data.

for ideal reverberation $\left(\sigma_{i}=\sigma_{j}=\sigma_{k}\right)$.

TABLE I

SUMMARY STATISTICS FOR $A$ AND $A^{\prime}$

\begin{tabular}{|r|r|r||r|r|r|}
\hline Parameter & $A$ & $A^{\prime}$ & Parameter & $A$ & $A^{\prime}$ \\
\hline Mean & 0.541 & 0.460 & 5\% Quantile & 0.176 & 0.144 \\
Median & 0.563 & 0.455 & 95\% Quantile & 0.826 & 0.809 \\
Std. Dev. & 0.202 & 0.196 & Skewness & -0.366 & 0.194 \\
Variance & 0.041 & 0.038 & Kurtosis & 2.317 & 2.599 \\
\hline
\end{tabular}

To compute quantiles that yield the lower and upper boundaries $\xi_{ \pm}$of symmetric $\eta \%$-confidence intervals for $A$, an approximate fit to $F_{A}^{-1}(y)$ at $y_{ \pm} \triangleq(1 \pm \eta / 100) / 2$ yields $\xi_{ \pm} \equiv F_{A}^{-1}\left[y_{ \pm}(\eta)\right]$ as

$$
\xi_{ \pm} \simeq 0.378 \exp \left(0.835 y_{ \pm}\right)-0.309 \exp \left(-6.173 y_{ \pm}\right) .
$$

For $\xi_{-} \simeq 0$ and $\xi_{+} \simeq 1$, a more accurate fit is e.g. $F_{A}^{-1}(y) \simeq$ $\sum_{i=0}^{9} \alpha_{i} y^{i}$ with $\alpha_{9}=532.2, \alpha_{8}=-2367, \alpha_{7}=4452, \alpha_{6}=$ $-4610, \alpha_{5}=2874, \alpha_{4}=-1109, \alpha_{3}=264.2, \alpha_{2}=-38.50$, $\alpha_{1}=4.205, \alpha_{0}=0.0365$. Similarly, $\xi_{ \pm}^{\prime} \equiv F_{A^{\prime}}^{-1}\left[y_{ \pm}(\eta)\right]$ for $A^{\prime}$ follows as

$$
\xi_{ \pm}^{\prime} \simeq 0.234 \exp \left(1.285 y_{ \pm}\right)-0.203 \exp \left(-13.040 y_{ \pm}\right) .
$$

A more accurate fit is $F_{A^{\prime}}^{-1}(y) \simeq \sum_{i=0}^{9} \alpha_{i}^{\prime} y^{i}$ with $\alpha_{9}^{\prime}=400.6$, $\alpha_{8}^{\prime}=-1754, \alpha_{7}^{\prime}=3251, \alpha_{6}^{\prime}=-3329, \alpha_{5}^{\prime}=2066$, $\alpha_{4}^{\prime}=-800.9, \alpha_{3}^{\prime}=193.9, \alpha_{2}^{\prime}=-29.13, \alpha_{1}^{\prime}=3.331$, $\alpha_{0}^{\prime}=0.0309$. Plots of the exact $\xi_{ \pm}^{(\prime)}$ and these approximations are shown in Fig. 9. While the confidence intervals for $A_{i j}$ and $A^{(\prime)}$ are fairly wide, those for their sample averages are much more narrowly localized [13].

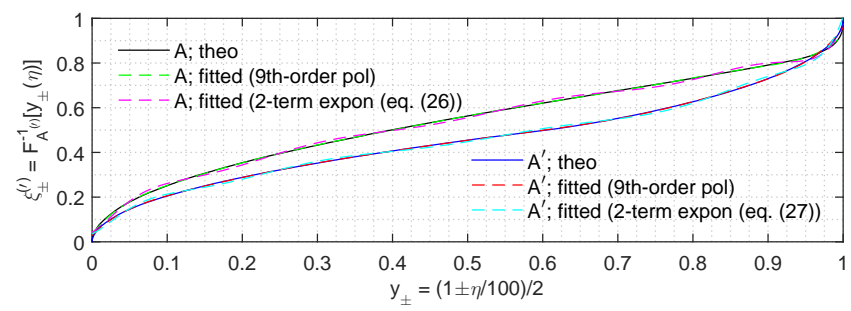

Fig. 9. Boundaries $\xi_{ \pm}$or $\xi_{ \pm}^{\prime}$ of $\eta \%$-confidence intervals for $A$ (upper curves) or $A^{\prime}$ (lower curves), respectively: exact theoretical values $F_{A^{(\prime)}}^{-1}\left[y_{ \pm}(\eta)\right]$ (solid black and blue), 9th-order polynomial approximations $\sum_{i=0}^{A_{0}} \alpha_{i}^{(\prime)} y_{ \pm}^{i}$ (dashed green and red), and two-term exponential series approximations eq (26) (dashed magenta) and eq. (27) (dashed cyan)

\section{INCOMPLETE STIRRING (DIRECT ILLUMINATION)}

\section{A. Partially Stirred Fields Resulting from Line-of-Sight Cou- pling or Diffractive Coupling}

Direct illumination, i.e., line-of-sight (LoS) propagation and coupling in MT/MSRCs can often be largely (but not completely) avoided by a proper choice of the relative orientation of transmitting and receiving antennas with reference to their radiation patterns. However, LoS coupling to a transmitting or receiving EUT may be more difficult to avoid, particularly for unintentional radiators or receptors with an unknown 'antenna' pattern for emissions. Moreover, fields from an antenna confined to a finite sized cavity do not satisfy the Sommerfeld radiation condition. Consequently, the coupling between an antenna and its shielded environment affects the radiation and near-field emission and their spatial patterns. At low frequencies, the patterns are typically wide (quasiisotropic radiation and reception), making it difficult to avoid LoS coupling completely. At high frequencies, the patterns for a typical in situ EUT are more directive but often highly irregular and nonintuitive in their angular variation, i.e., quasirandom. As a result, significant coupling may occur in certain directions, locations and relative orientations. Furthermore, particularly at low frequencies, diffraction by the edges of EM shields constitutes an additional source of EM coupling. Finally, because of the time-varying nature of MT/MSRCs, the direct EM coupling may itself fluctuate and may not be completely avoidable for all configurations (set of boundary conditions) during the stir process.

1) General Levels of Direct Illumination: Direct illumination causes at least one (spatial) component $E_{i}$ - say its inphase field component $E_{i}^{\|}$, without loss of generality - to exhibit a nonzero mean, i.e., a deterministic finite value $\mu_{E_{i}^{\|}}$ [3]. The quadrature component $E_{i}^{\perp}$ may have the same or other mean value $\mu_{E_{i}^{\perp}}$, irrespective of whether the random field is circular or not. The mean of $E_{i}=E_{i}^{\|}+\mathrm{j} E_{i}^{\perp}$ is $\mu_{E_{i}}=\mu_{E_{i}^{\|}}+\mathrm{j} \mu_{E_{i}^{\perp}}$, by definition [14]. Consequently, $X_{i}$ is 
now distributed as a noncentral $\chi_{2}^{2}$ pdf [15, p. 343], i.e.,

$$
\begin{aligned}
& f_{X_{i}}\left(x_{i}\right)=\left(2 \sqrt{\pi} \sigma_{i}\right)^{-1} \exp \left[-\left(\tau_{i}+\frac{x_{i}}{2 \sigma_{i}}\right)\right] \\
& \times \sum_{m=0}^{\infty} \frac{\Gamma\left(m+\frac{1}{2}\right)}{m !(2 m) !}\left(\frac{2 \tau_{i} x_{i}}{\sigma_{i}}\right)^{m} \\
& =\sum_{m=0}^{\infty} \frac{\tau_{i}^{m} \exp \left(-\tau_{i}\right)}{m !} \frac{\left[x_{i} /\left(2 \sigma_{i}\right)\right]^{m} \exp \left[-x_{i} /\left(2 \sigma_{i}\right)\right]}{2(m !) \sigma_{i}},
\end{aligned}
$$

with noncentrality parameter

$$
\tau_{i} \triangleq \frac{\left|\mu_{E_{i}} / \sigma_{E_{i}}\right|^{2}}{2}=\frac{\left(\mu_{E_{i}^{\|}} / \sigma_{E_{i}^{\|}}\right)^{2}+\left(\mu_{E_{i}^{\perp}} / \sigma_{E_{i}^{\perp}}\right)^{2}}{2},
$$

in which $\sigma_{E_{i}}$ and $\sigma_{i}$ represent the reference standard deviation of $E_{i}$ and $\left|E_{i}\right|^{2}$, respectively, in the absence of direct illumination, which are hence still related via (4). In each term of (29), the first ratio $\tau_{i}^{m} \exp \left(-\tau_{i}\right) /(m !)$ corresponds to the pdf of a discrete Poisson process with parameter $\tau_{i}$. The second ratio is a central $\chi_{2(m+1)}^{2}$ pdf, i.e., a gamma pdf with scale parameter $m+1$. Hence the noncentral $\chi_{2}^{2}$ can be conceived as the superposition of an infinite number of Poisson-weighted arrival processes. With the aid of

$$
\int x^{n} \exp (-x) \mathrm{d} x=\exp (-x) \sum_{\ell=0}^{n} \frac{(-1)^{\ell-1} n ! x^{n-\ell}}{(n-\ell) !}+C,
$$

the mean and variance of $\left|E_{i}\right|^{2}$ follow ${ }^{4}$ for arbitrary $\tau_{i}$ as

$$
\mu_{X_{i}}=2\left(1+\tau_{i}\right) \sigma_{E_{i}}^{2}, \quad \sigma_{X_{i}}=2 \sqrt{1+2 \tau_{i}} \sigma_{E_{i}}^{2} .
$$

Thus, the relative level of fluctuation $\nu_{X_{i}}=\sigma_{X_{i}} / \mu_{X_{i}}$, decreases from unity as $\tau_{i}$ increases, whence the sample coefficient $n_{i} \triangleq s_{X_{i}} / m_{X_{i}}$ may be interpreted as measure of direct illumination. More generally, $\nu_{i}$ is indicative of an imperfectly stirred ("understirred") field for the direction $i$ at $\underline{r}$, i.e., of any deterministic polarization bias in this direction.

Whether a corresponding statistical polarization bias exists - possibly at a different scale of fluctuation - must be assessed through a study of the anisotropy for the stirred vector field (cf. Sec. III). Apart from direct illumination, this is also possible in non-spherically or non-cubically symmetric cavities when the frequency of excitation is too low in order for a sufficient number of contributing modes to coexist, i.e., below the 'lowest' usable frequency of the chamber [1]. At relatively low frequencies, the existence of only few modes with different modal $Q$-factors (nonergodic regime) yields a strongly anisotropic vector field. However, at these low frequencies, the present analysis may not be applicable, irrespective of any direct illumination, because the assumption of a noncentral $\chi_{2}^{2}$ pdf still implies that the centered ("dc-filtered", debiased) field has a Gauss normal distribution. Such is guaranteed only if the number of modes is sufficiently large.

The expressions (32) that yield $\nu_{X_{i}}$ may be used in a moment method estimation of $\tau_{i}$ as

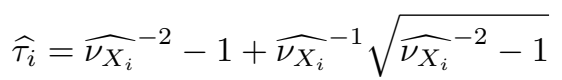

${ }^{4}$ See e.g., [16, eqs. (8) and (9)] for expressions for $\mu_{\left|E_{i}\right|}$ and $\sigma_{\left|E_{i}\right|}^{2}$. where $\widehat{\nu_{X_{i}}} \triangleq \widehat{\sigma_{X_{i}} / \mu_{X_{i}}}=\sqrt{(n-1) / n} n_{i}$ is an unbiased estimator of $\nu_{X_{i}}$, with $n_{i} \triangleq s_{X_{i}} / m_{X_{i}}$ for a sample of size $n$.

2) Residual Direct Illumination: For sufficiently small noncentralities, viz., $\tau_{i} x_{i} / \sigma_{i} \ll 1$, one may approximate ${ }^{5}$ (28) to first order by retaining its two leading terms only, i.e.,

$$
\begin{aligned}
f_{X_{i}}\left(x_{i}\right) & =\frac{1}{2 \sigma_{i}} \exp \left[-\left(\tau_{i}+\frac{x_{i}}{2 \sigma_{i}}\right)\right] \sum_{m=0}^{\infty} \frac{\left(\tau_{i} x_{i} / \sigma_{i}\right)^{m}}{2^{m}(m !)^{2}} \\
& \simeq \frac{1}{2 \sigma_{i}}\left(1+\frac{\tau_{i} x_{i}}{2 \sigma_{i}}\right) \exp \left[-\left(\tau_{i}+\frac{x_{i}}{2 \sigma_{i}}\right)\right]
\end{aligned}
$$

In view of the dominant exponential decay, (35) has been found to remain a reasonable approximation for (28) up to intermediate values of $\tau_{i} x_{i} / \sigma_{i}$ of order unity [7, Fig. 4].

For relatively low intensity of the direct illumination and otherwise isotropic stirring performance $\left(\sigma_{i}=\sigma_{j} \triangleq \sigma\right.$, assuming temporarily $\sigma \equiv 1$ for simplicity), $f_{X_{i}}\left(x_{i}\right)$ and $f_{X_{j}}\left(x_{j}\right)$ can be replaced by their two-term approximations (35). Following the method for calculating $A_{i j}$ in the nonLoS scenario in the Appendix, the pdf is obtained after straightforward algebra as

$$
f_{A_{i j}}\left(a_{i j}\right)=\frac{1}{2}\left(1+\frac{\tau_{i}-\tau_{j}}{1+\tau_{i}+\tau_{j}} a_{i j}\right), 1 \leq a_{i j} \leq 1 .
$$

The corresponding cdf is

$$
F_{A_{i j}}\left(a_{i j}\right)=\frac{1}{2}\left(a_{i j}+1\right)+\frac{1}{4} \frac{\tau_{i}-\tau_{j}}{1+\tau_{i}+\tau_{j}}\left(a_{i j}^{2}-1\right) .
$$

This pdf and cdf are shown in Fig. 10 for selected values of $\tau_{i}$ and $\tau_{j}$. The mean and standard deviation of $A_{i j}$ are now

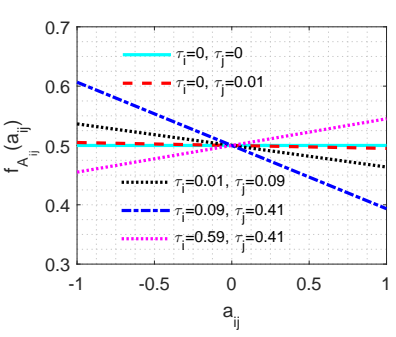

(a)

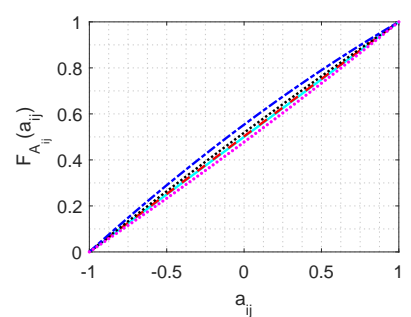

(b)
Fig. 10. (a) Pdfs $f_{A_{i j}}\left(a_{i j}\right)$ and (b) corresponding cdfs $F_{A_{i j}}\left(a_{i j}\right)$ (same line type and color code) for selected levels of noncentralities $\tau_{i}$ and $\tau_{j}$ and assuming statistically isotropic stirring performance $\left(\sigma_{i}=\sigma_{j}\right)$.

$$
\begin{aligned}
\mu_{A_{i j}} & =\frac{\tau_{i}-\tau_{j}}{3\left(1+\tau_{i}+\tau_{j}\right)} \\
\sigma_{A_{i j}} & =\frac{\sqrt{3\left(1+\tau_{i}+\tau_{j}\right)^{2}-\left(\tau_{i}-\tau_{j}\right)^{2}}}{3\left(1+\tau_{i}+\tau_{j}\right)}
\end{aligned}
$$

which are shown as a function of $\tau_{i}$ and $\tau_{j}$ in Fig. 11 .

Comparison of Fig. 1(a) and Fig. 10(a) shows that the pdf (36) is nearly trapezoidal, i.e., having a nearly linear dependence on $a_{i j}$ when $\sigma_{j} / \sigma_{i} \gtrsim 1$ and $\tau_{i}=\tau_{j}=0$. This pdf is similar to that for small positive $\tau_{j}-\tau_{i}$ with

\footnotetext{
${ }^{5}$ Note that $X_{i}$ itself can take small as well as large values, hence the exponential function should not be expanded even for small noncentralities.
} 
$\sigma_{i}=\sigma_{j}=1$. Explicitly, comparison of (5) and (36) suggests that correspondence occurs when

$$
\frac{\sigma_{j}-\sigma_{i}}{\sigma_{j}+\sigma_{i}}=\frac{\tau_{j}-\tau_{i}}{2\left(1+\tau_{j}+\tau_{i}\right)}, \quad\left(\sigma_{j} / \sigma_{i} \simeq 1, \tau_{i, j} \ll 1\right) .
$$

Thus, for field fluctuations and bias levels whose parameter values satisfy (40), spatial stir anisotropy and unequal direct illumination are indistinguishable in their effect on $f_{A_{i j}}\left(a_{i j}\right)$.

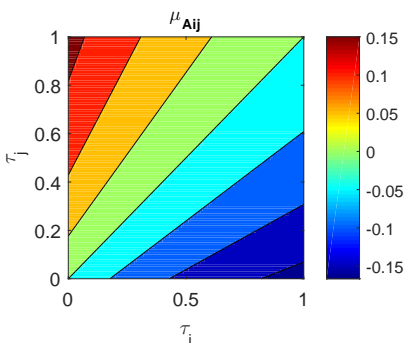

(a)

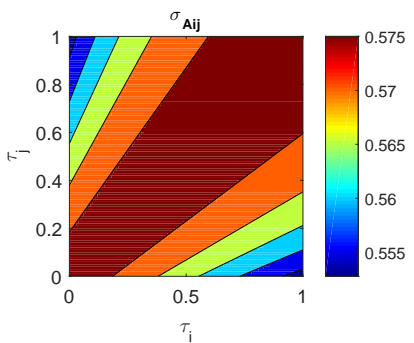

(b)
Fig. 11. (a) Mean and (b) standard deviation of $A_{i j}$ as a function of direct illumination (noncentralities $\tau_{i, j}$ ), assuming isotropic stirring quality $\left(\sigma_{i}=\right.$ $\left.\sigma_{j}\right)$.

B. General Imperfect Stirring: Stirring Anisotropy in Conjunction with Direct Illumination (Line-of-Sight Coupling)

The results of Secs. III and IV-A can now be collated to characterize the general case of residual direct illumination (nonzero deterministic field content $\mu_{i}$ ) in combination with spatially anisotropic stirring (arbitrary different values of $\sigma_{i}$ and $\sigma_{j}$ ). Using again the two-term approximation (35), now with $\sigma_{i, j} \neq 1$ in general, the pdf and cdf follow after cumbersome but straightforward algebra as

$$
\begin{gathered}
f_{A_{i j}}\left(a_{i j}\right)=\frac{1}{c} \frac{\left(c_{+}+2 d_{+}\right)+\left(c_{-}+2 d_{-}\right) a_{i j}}{\left(c_{+}+c_{-} a_{i j}\right)^{3}}, \\
F_{A_{i j}}\left(a_{i j}\right)=\frac{1}{2\left(c_{-}\right)^{2} c}\left[\frac{\beta c_{+}-\alpha c_{-}}{\left(c_{+}+c_{-} a_{i j}\right)^{2}}\right. \\
\left.-\frac{2 \beta}{\left(c_{+}+c_{-} a_{i j}\right)}+\frac{(\alpha-2 \beta) c_{-}+\beta c_{+}}{\left(c_{+}-c_{-}\right)^{2}}\right]
\end{gathered}
$$

valid for $-1 \leq a_{i j} \leq 1$, where

$$
\begin{aligned}
c_{ \pm} \triangleq \sigma_{i}^{-1} \pm \sigma_{j}^{-1}, & d_{ \pm} \triangleq \frac{\tau_{i}}{\sigma_{i}} \pm \frac{\tau_{j}}{\sigma_{j}}, \\
\alpha \triangleq c_{+}+2 d_{+}, & \beta \triangleq c_{-}+2 d_{-},
\end{aligned}
$$

and with normalization constant

$$
\begin{aligned}
c \triangleq \frac{\sigma_{i} \sigma_{j}}{2}\{1+ & {\left[1+\frac{1}{2}\left(\sigma_{r}+\sigma_{r}^{-1}\right)\right] \frac{d_{+}}{c_{+}} } \\
& \left.+\left[1-\frac{1}{2}\left(\sigma_{r}+\sigma_{r}^{-1}\right)\right] \frac{d_{-}}{c_{-}}\right\} .
\end{aligned}
$$

This pdf and cdf are shown in Fig. 12 for selected values of $\tau_{j}$ and $\sigma_{j}$, for the case of a standardized non-LoS random field for $E_{i}$, i.e., for $\tau_{i}=0$ and $\sigma_{i}=1$. It can be verified that (41) and (42) reduce to the pdf and cdf for the particular cases of unbiased fields $\left(\tau_{i}=\tau_{j}=0, \sigma_{i} \neq \sigma_{j}\right.$; eqs. (5) and (6)) and biased normalized fields $\left(\tau_{i}, \tau_{j} \neq 0, \sigma_{i}=\sigma_{j}=1\right.$; eqs. (36) and (37)).

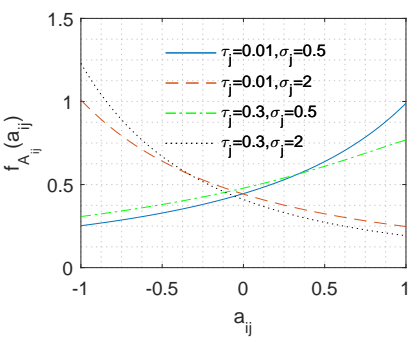

(a)

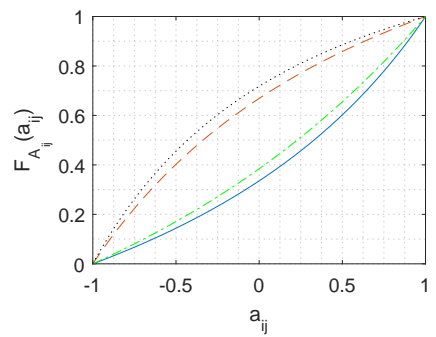

(b)
Fig. 12. (a) Pdfs $f_{A_{i j}}\left(a_{i j}\right)$ and (b) corresponding cdfs $F_{A_{i j}}\left(a_{i j}\right)$ (same line type and color code as in figure (a)) for selected levels of noncentrality $\tau_{j}$ and stir anisotropy $\sigma_{j}$, assuming $\tau_{i}=0$ and $\sigma_{i}=1$.

\section{Field Anisotropy in Undermoded Chambers}

A fundamental assumption held so far (cf. Sec. III-A) is that the underlying complex fields $E_{i}$ in the definitions of $A_{i j}$ and $A^{(\prime)}$ exhibit independent and identical ensemble circular Gaussian pdfs. Near or below the LUF [1], this assumption may be unsustainable. In that case, a characterization based on sampling circular, elliptic [17], or even more general [18] joint pdfs for $E_{i}$ and $E_{j}$ may be appropriate. A complete generalization involves additional parameters of the distribution, each requiring individual estimation (e.g., using Bayesian inference), and the characterization of the dependence structure between $E_{i}$ and $E_{j}$, e.g., using copulas [18]. Here, we simply show how small sample sizes affect the cdf of $A_{i j}$ and $A$ by using sampling distributions ${ }^{6}$ of $\left|E_{i}\right|^{2}$ for circular Gaussian $E_{i}$ [19] and manifested by a finite number of degrees of freedom $\mathcal{N}_{i}$ for $E_{i}$.

Fig. 13 shows $F_{A_{i j}}\left(a_{i j}\right)$ and $F_{A}(a)$ at selected values of $\mathcal{N}_{i}$ for ensemble isotropic fields $\left(\sigma_{i}=\sigma_{i}=\sigma_{k}\right)$. An inverted sigmoid shape of $F_{A_{i j}}\left(a_{i j}\right)$ is observed - corresponding to a quasi-uniform $f_{A_{i j}}\left(a_{i j}\right)$ with warped edges that tends toward a U-shaped pdf (not shown) - as well as an increase of $\langle A\rangle$ for decreasing $\mathcal{N}_{i}$. The distributions were obtained numerically based on MC simulation, using an inverse probability integral transform for generating triplets of mutually independent Bessel $K$ distributed samples $\left\{\left|e_{i, n}\right|^{2},\left|e_{j, n}\right|^{2},\left|e_{k, n}\right|^{2}\right\}_{n=1}^{10,000}$, i.e., with $\left|e_{i, n}\right|^{2}=F_{U_{i}}^{-1}\left(u_{i, n}\right)$ for each $(n$ th) sample, where $U_{i}$ is uniformly distributed on $[0,1]$, with [20, eq. (25)]

$$
F_{\left|E_{i}\right|^{2}}\left(\left|e_{i}\right|^{2}\right)=1-\frac{\left(\sqrt{\frac{\left|e_{i}\right|^{2}}{\sigma_{E_{i}}^{2}}}\right)^{\mathcal{N}_{i}} K_{\mathcal{N}_{i}}\left(\sqrt{\frac{\left|e_{i}\right|^{2}}{\sigma_{E_{i}}^{2}}}\right)}{2^{\mathcal{N}_{i}-1} \Gamma\left(\mathcal{N}_{i}\right)} .
$$

Here, $K_{\mathcal{N}_{i}}(\cdot)$ is the modified Bessel function of the second kind and order $\mathcal{N}_{i}$, while $\Gamma(\cdot)$ denotes the complete gamma function.

\section{CONCLUSION}

In this paper, probability distributions of planar and total (vector) field anisotropy coefficients $A_{i j}, A$ and $A^{\prime}$ were derived - analytically in closed form, computationally through

\footnotetext{
${ }^{6}$ Since the standard deviations of $\left|E_{i, j}\right|^{2}$ do not enter the definition (2), the use of Student $t$ sampling pdfs would overestimate the effect of finite sized sampling; cf. [19, Fig. 2] for a comparison.
} 


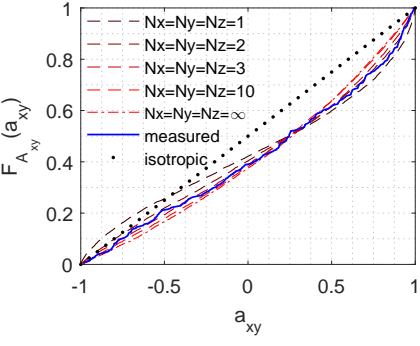

(a)

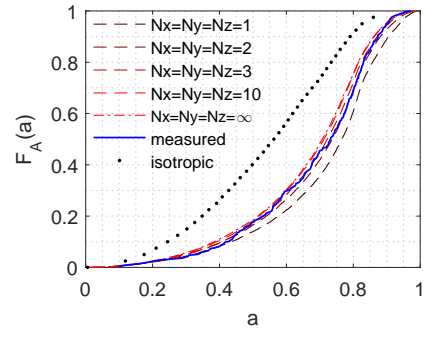

(b)
Fig. 13. Sampling based (dashed) vs. empirical (solid) cdfs of $A_{x y}$ and $A$ based on Bessel $K$ sampling cdfs for $\left|E_{x, y, z}\right|^{2}$ with selected triplet values of NDoFs $\mathcal{N}_{x}=\mathcal{N}_{y}=\mathcal{N}_{z}$ as indicated, based on measured fields at $d=$ $0.075 \lambda$ with sample standard deviations $s_{x, y, z}$. Ensemble cdfs (dash dotted) and ideal isotropy cdfs (black dots) are shown for reference.

numerical quadrature, and via Monte Carlo simulation - and compared with empirical distributions for measured data. The particular scenarios investigated include anisotropic stir performance (eqs. (5), (17), (24)), ideal statistically isotropic reverberation (eq. (7)), residual direct illumination (line-ofsight coupling) (eq. (36)), and the combination of the latter two cases (eq. (41)).

Unlike the field uniformity metric in [1, Appendix B], a field anisotropy coefficient quantifies the separate local statistical anisotropy aspect of the field nonuniformity, without mixing it with (nonlocal) inhomogeneity of the field. More general scenarios were described in Sec. I, whereby specific types of anisotropic random fields with selected aspect ratios $\sigma_{i}: \sigma_{j}: \sigma_{k}$, defined by (4), may act as reference fields.

The analysis incorporates imperfect reverberation to the extent that each Cartesian (spatial) field component is assumed to exhibit a complex circular normal distribution with mean and standard deviation that can differ between components. Per component, however, ideal circular Gaussianity of its inphase and quadrature contributions was assumed [21]. The methods and results can be extended to more general elliptic fields and their components, along the lines of [2], [17], and to include inter-Cartesian correlation or higher-order interdependence. Departures from circularity lead to increased kurtosis of the marginal pdfs, including a heavier tail of the pdf. The effect of the latter was investigated here through the use of sampling pdfs for fields and intensities, showing qualitatively the effects on the cdfs of $A_{i j}$ and $A$.

While the focus was on statistical anisotropy of the local field, the concepts, definitions, and analysis of $A_{i j}$ and $A$ can be extended mutatis mutandis to statistical field inhomogeneity, based on corresponding two- and multi-point field inhomogeneity coefficients $I_{k \ell}$ at $\underline{r}_{k}$ and $\underline{r}_{\ell}$ (for an arbitrary polarization direction $i$ of the field) and their combination to two-point total-field inhomogeneity [7]. Thus, a general framework and methodology is obtained wherein field anisotropy and homogeneity can both be assessed individually or in combination. For some EMC testing in MT/MSRCs, e.g., immunity of electrically small EUTs, it is conceivable that a metric of the field uniformity based on mixed anisotropy and inhomogeneity performance (as in [1, Appendix], but a m may often be too stringent or otherwise unsuitable for the application at hand. For such cases, an appropriately weighted and adaptive mixed nonuniformity metric $N_{i j, k \ell}$ can be devised, involving both $A_{i j}$ and $I_{k \ell}$ for different locations and spatial directions of polarization, and $\mathrm{I} / \mathrm{Q}$ phasors.

Apart from their use in the evaluation and improved operation of MT/MSRCs, the methods and results also enable an improved probabilistic field characterization in various EMC test scenarios and applications. These include stochastic boundary-value problems involving metallic walls or interfaces with dielectric bodies, electrically large EUTs.

\section{REFERENCES}

[1] Joint Task Force IEC CISPR/A-SC77B: IEC 61000-4-21 Electromagnetic Compatibility (EMC) - Part 4-21: Testing and Measurement Techniques - Reverberation Chamber Test Methods, International Electrotechnical Commission, 2nd ed., Jan. 2011.

[2] R. Serra and F. Canavero, "Bivariate statistical approach for 'good-butimperfect' electromagnetic reverberation," IEEE Trans. Electromagn. Compat., vol. 53, no. 3, pp. 554-561, Aug. 2011.

[3] J. G. Kostas and B. Boverie, "Statistical model for a mode-stirred chamber," IEEE Trans. Electromagn. Compat., vol. 33, no. 4, pp. 366370, Nov. 1991.

[4] P. Corona, G. Ferrara, and M. Migliaccio, "Reverberating chambers as sources of stochastic electromagnetic fields," IEEE Trans. Electromagn. Compat., vol. 38, no. 3, pp. 348-356, Aug. 1996.

[5] L. R. Arnaut and P. D. West, "Electric field probe measurements in the NPL untuned stadium reverberation chamber," NPL Report, CETM 13, Sep. 1999, pp. 1-304.

[6] C. Lemoine, P. Besnier, and M. Drissi, "Estimating the effective sample size to select independent measurements in a reverberation chamber," IEEE Trans. Electromagn. Compat., vol. 50, no. 2, pp. 227-236, May 2008.

[7] L. R. Arnaut, "Field anisotropy, field inhomogeneity and polarization bias in imperfect reverberation chambers," NPL Techn. Note, R981120, Nov. 1998, pp. 1-65

[8] L. R. Arnaut, "Operation of electromagnetic reverberation chambers with wave diffractors at relatively low frequencies," IEEE Trans. Electromagn. Compat., vol. 32, no. 4, pp. 637-653, Nov. 2001.

[9] L. R. Arnaut and P. D. West, "Evaluation of the NPL untuned stadium reverberation chamber using mechanical and electronic stirring techniques," NPL Report, CEM 11, Nat. Phys. Lab., Teddington, U.K., ISSN 1369-6742, Aug. 1998.

[10] D. Kelker, "Distribution theory of spherical distributions and a locationscale parameter generalization," Sankhyā A, vol. 32, pp. 419-430, 1970

[11] R. J. Pirkl and K. A. Remley, "Experimental evaluation of the statistica isotropy of a reverberation chambers plane-wave spectrum," IEEE Trans. Electromagn. Compat., vol. 56, no. 3, pp. 498-509, Jun. 2014.

[12] T. H. Lehman, "A statistical theory of electromagnetic fields in complex cavities," USAF Phillips Laboratory Interaction Note, No. 494, 1993.

[13] L. R. Arnaut, R. Serra, and P. D. West, "Validating reverberation chamber performance based on assessment of field anisotropy," to be presented at EMC Europe 2014 Int. Symp. Electromagn. Compat., paper 134, Wroclaw, Poland, Sep. 2016.

[14] A. Papoulis, Probability, Random Variables, and Stochastic Processes 2nd ed., McGraw-Hill: New York, NY, Ch. 5, p. 107, 1984.

[15] M. Fisz, Probability Theory and Mathematical Statistics. 3rd ed., Wiley: NY, Ch. 9, 1963

[16] P. Corona, G. Ferrara, and M. Migliaccio, "Reverberating chamber electromagnetic field in presence of an unstirred component," IEEE Trans. Electromagn. Compat., vol. 42, no. 2, pp. 111-115, May 2000.

[17] L. R. Arnaut, "Elliptic stochastic fields in reverberation chambers," IEEE Trans. Electromagn. Compat., vol. 58, no. 1, pp. 11-21, Feb. 2016.

[18] L. R. Arnaut, "Copulas, outliers and rogue states of nonelliptic fields and energy in electromagnetic reverberation," IEEE Trans. Electromagn. Compat., vol. 58, no. 2, pp. 371-384, Apr. 2016.

[19] L. R. Arnaut, "Sampling distributions of random electromagnetic fields in mesoscopic or dynamical systems," Phys. Rev. E, vol. 80, no. 3 03661, Sep. 2009

[20] L. R. Arnaut, "Limit distributions for imperfect electromagnetic reverberation," IEEE Trans. Electromagn. Compat., vol. 45, no. 2, pp. 357377, May 2003. 
[21] L. R. Arnaut, "Compound exponential distributions for undermoded reverberation chambers," IEEE Trans. Electromagn. Compat., vol. 44, no. 3, pp. 442-457, May 2002.

\section{APPENDIX}

\section{A. Anisotropic Stirring in Absence of Direct Illumination}

The pdf of the anisotropy coefficient $A_{i j}$ is obtained in two steps. First, define $X_{i} \triangleq\left|E_{i}\right|^{2}$ and perform a transformation of variables as

$$
g:\left(X_{i}, X_{j}\right) \rightarrow\left(U_{1}=X_{i}+X_{j}, U_{2}=X_{i}-X_{j}\right)
$$

which maps the domain of $g$ in the $o x_{i} x_{j}$-plane, i.e., $\left(x_{i} \geq 0\right.$, $\left.x_{j} \geq 0\right)$ in the $o x_{i} x_{j}$-plane, to the wedge $\left(\left|u_{2}\right| \leq u_{1}\right)$ in the $o u_{1} u_{2}$-plane. The joint pdf of $U_{1}$ and $U_{2}$ is

$$
\begin{aligned}
& f_{U_{1}, U_{2}}\left(u_{1}, u_{2}\right)=f_{X_{i}, X_{j}}\left[g_{1}^{-1}\left(u_{1}, u_{2}\right), g_{2}^{-1}\left(u_{1}, u_{2}\right)\right] \cdot\left|J_{g}\right| \\
& =\left(8 \sigma_{i} \sigma_{j}\right)^{-1} \exp \left[-\frac{u_{1}}{4}\left(\sigma_{i}^{-1}+\sigma_{j}^{-1}\right)\right. \\
& \left.-\frac{u_{2}}{4}\left(\sigma_{i}^{-1}-\sigma_{j}^{-1}\right)\right] \text {, } \\
& 0 \leq u_{1}<+\infty,\left|u_{2}\right| \leq u_{1}
\end{aligned}
$$

where $J_{g} \triangleq \operatorname{det}\left[\frac{\partial g^{-1}\left(U_{1}, U_{2}\right)}{\partial\left(X_{1}, X_{2}\right)}\right]=-1 / 2$ is the Jacobian of the inverse transformation $g^{-1}$, the latter's multiplicity being 1 . In arriving at (48), use was made of the assumption that $E_{i}$ and $E_{j}$ and, hence, $X_{i}$ and $X_{j}$ are statistically independent $\left(f_{X_{i} X_{j}}=f_{X_{i}} f_{X_{j}}\right)$. The marginal pdfs are obtained as

$$
\begin{aligned}
& f_{U_{1}}\left(u_{1}\right)=\int_{-u_{1}}^{u_{1}} f_{U_{1}, U_{2}}\left(u_{1}, u_{2}\right) \mathrm{d} u_{2} \\
&=\left[2\left(\sigma_{j}-\sigma_{i}\right)\right]^{-1}\left[\exp \left(-\frac{u_{1}}{2 \sigma_{j}}\right)-\exp \left(-\frac{u_{1}}{2 \sigma_{i}}\right)\right] \\
&=\frac{\exp \left[-\frac{u_{1}}{4}\left(\sigma_{i}^{-1}+\sigma_{j}^{-1}\right)\right]}{\left(\sigma_{j}^{-1}-\sigma_{i}^{-1}\right)} \sinh \left[\frac{u_{1}}{4}\left(\sigma_{i}^{-1}-\sigma_{j}^{-1}\right)\right] \\
& f_{U_{2}}\left(u_{2}\right)=\int_{\left|u_{2}\right|}^{\infty} f_{U_{1}, U_{2}}\left(u_{1}, u_{2}\right) \mathrm{d} u_{1} \\
&=\left[2\left(\sigma_{i}+\sigma_{j}\right)\right]^{-1} \exp \left(-\frac{u_{2}+\left|u_{2}\right|}{4 \sigma_{i}}+\frac{u_{2}-\left|u_{2}\right|}{4 \sigma_{j}}\right), \\
& u_{2} \leq\left|u_{1}\right| .
\end{aligned}
$$

$$
\begin{aligned}
f_{U_{2}}\left(u_{2}\right)= & \frac{1}{4}\left(1+\frac{\tau_{i}+\tau_{j}}{2}+\frac{\tau_{i}-\tau_{j}}{4} u_{2}+\frac{\tau_{i}+\tau_{j}}{4}\left|u_{2}\right|\right) \\
& \times \exp \left[-\left(\tau_{i}+\tau_{j}+\frac{\left|u_{2}\right|}{2}\right)\right]
\end{aligned}
$$

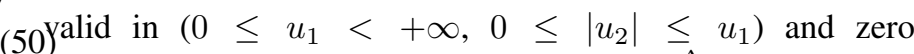
otherwise. With the same transformations $V_{1} \triangleq U_{2} / U_{1} \equiv A_{i j}$, $V_{2} \triangleq U_{2}$, we obtain the pdf of $A_{i j}$, after some straightforward calculation and final normalization, as

$f_{U_{1}}\left(u_{1}\right)=\frac{u_{1}}{4 \sigma^{2}} \exp \left(-\frac{u_{1}}{2 \sigma}\right), f_{U_{2}}\left(u_{2}\right)=(4 \sigma)^{-1} \exp \left(-\frac{\left|u_{2}\right|}{2 \sigma}\right)$,

$$
f_{A_{i j}}\left(a_{i j}\right)=\frac{1}{2}\left(1+\frac{\tau_{i}-\tau_{j}}{1+\tau_{i}+\tau_{j}} a_{i j}\right), \quad-1 \leq a_{i j} \leq 1 \text {. }
$$

where the former is a $\chi_{4}^{2}\left(u_{1}\right)$ pdf for the sum of two independent normalized $\chi_{2}^{2}$-distributed field intensities.

Next, a second transformation of variables is defined by

$$
h:\left(U_{1}, U_{2}\right) \rightarrow\left(V_{1}=U_{2} / U_{1}, V_{2}=U_{2}\right)
$$

where $V_{2}$ is a dummy variate. This transforms the domain $\left(\left|u_{2}\right| \leq u_{1}\right)$ of $h$ in the $o u_{1} u_{2}$-plane to $\left(0 \leq\left|v_{1}\right| \leq 1\right.$, $\left.v_{1} v_{2} \geq 0\right)$ in the $o v_{1} v_{2}$-plane. The latter consists of two

\section{Incomplete Anisotropic Stirring (Direct Illumination)}

For the general imperfect stirring case, stirring anisotropy and incomplete stirring are combined and result in

$$
\begin{aligned}
f_{X_{i}}\left(x_{i}\right) & =\frac{1}{2 \sigma_{i}} \exp \left[-\left(\tau_{i}+\frac{x_{i}}{2 \sigma_{i}}\right)\right] \sum_{m=0}^{\infty} \frac{\left(\tau_{i} x_{i} / \sigma_{i}\right)^{m}}{2^{m}(m !)^{2}} \\
& \simeq \frac{1}{2 \sigma_{i}}\left(1+\frac{\tau_{i} x_{i}}{2 \sigma_{i}}\right) \exp \left[-\left(\tau_{i}+\frac{x_{i}}{2 \sigma_{i}}\right)\right] .
\end{aligned}
$$


Now, $\tau_{i} \triangleq(1 / 2) \sum_{k=\|, \perp}\left(\mu_{i, k} / \sigma_{i, k}\right)^{2}$ is the standardized, (hence dimensionless) stirring bias for the polarization direction $i$ where $\sigma_{i, \|} \triangleq \sigma_{E_{i}^{\|}}$and $\sigma_{i, \perp} \triangleq \sigma_{E_{i}^{\perp}}$. The latter two equalities hold for circular complex Cartesian fields $\left(\sigma_{E_{i}^{\|}}=\sigma_{E_{i}^{\perp}}, \rho_{E_{i}^{\|}, E_{i}^{\perp}}=0\right)$.

The joint and marginal distributions for the auxiliary $U_{1}$ and $U_{2}$ follow, after lengthy but straightforward calculation, as

$$
\begin{aligned}
& f_{U_{1}, U_{2}}\left(u_{1}, u_{2}\right)=\frac{1}{8 \sigma_{i} \sigma_{j}}\left[1+\left(\frac{\tau_{i}}{\sigma_{i}}+\frac{\tau_{j}}{\sigma_{j}}\right) \frac{u_{1}}{4}+\left(\frac{\tau_{i}}{\sigma_{i}}-\frac{\tau_{j}}{\sigma_{j}}\right) \frac{u_{2}}{4}\right] \\
& \times \exp \left\{-\left[\tau_{i}+\tau_{j}+\left(\sigma_{i}^{-1}+\sigma_{j}^{-1}\right) \frac{u_{1}}{4}+\left(\sigma_{i}^{-1}-\sigma_{j}^{-1}\right) \frac{u_{2}}{4}\right]\right\}, \\
& f_{U_{1}}\left(u_{1}\right)=\frac{1}{\sigma_{j}-\sigma_{i}}\left[1+\frac{\frac{\tau_{i}}{\sigma_{i}}-\frac{\tau_{j}}{\sigma_{j}}}{\sigma_{i}^{-1}-\sigma_{j}^{-1}}+\left(\frac{\tau_{i}}{\sigma_{i}}+\frac{\tau_{j}}{\sigma_{j}}\right) \frac{u_{1}}{4}\right] \\
& \times \exp \left[-\left(\tau_{i}+\tau_{j}+\left(\sigma_{i}^{-1}+\sigma_{j}^{-1}\right) \frac{u_{1}}{4}\right)\right] \sinh \left[\left(\sigma_{i}^{-1}-\sigma_{j}^{-1}\right) \frac{u_{1}}{4}\right] \\
& -\frac{\frac{\tau_{i}}{\sigma_{i}}-\frac{\tau_{j}}{\sigma_{j}}}{\sigma_{i}^{-1}-\sigma_{j}^{-1}} \frac{u_{1}}{4} \exp \left[-\left(\tau_{i}+\tau_{j}+\left(\sigma_{i}^{-1}+\sigma_{j}^{-1}\right) \frac{u_{1}}{4}\right)\right] \cosh \left[\left(\sigma_{i}^{-1}-\sigma_{j}^{-1}\right) \frac{u_{1}}{4}\right] \\
& f_{U_{2}}\left(u_{2}\right)=\frac{1}{2 \sigma_{i} \sigma_{j}\left(\sigma_{i}^{-1}+\sigma_{j}^{-1}\right)}\left[1+\frac{\frac{\tau_{i}}{\sigma_{i}}+\frac{\tau_{j}}{\sigma_{j}}}{\sigma_{i}^{-1}+\sigma_{j}^{-1}}+\left(\frac{\tau_{i}}{\sigma_{i}}-\frac{\tau_{j}}{\sigma_{j}}\right) \frac{u_{2}}{4}+\left(\frac{\tau_{i}}{\sigma_{i}}+\frac{\tau_{j}}{\sigma_{j}}\right) \frac{\left|u_{2}\right|}{4}\right] \\
& \times \exp \left[-\left(\tau_{i}+\tau_{j}+\left(\sigma_{i}^{-1}-\sigma_{j}^{-1}\right) \frac{u_{2}}{4}+\left(\sigma_{i}^{-1}+\sigma_{j}^{-1}\right) \frac{\left|u_{2}\right|}{4}\right)\right],
\end{aligned}
$$

again valid inside $\left(0 \leq u_{1}<+\infty, 0 \leq\left|u_{2}\right| \leq u_{1}\right)$ while zero outside this region. With the same transformations $V_{1} \triangleq$ $U_{2} / U_{1} \equiv A_{i j}, V_{2} \triangleq U_{2}$, we obtain after some calculation $f_{A_{i j}}\left(a_{i j}\right)=\frac{1}{c} \frac{\left[\left(\sigma_{i}^{-1}+\sigma_{j}^{-1}\right)+2\left(\frac{\tau_{i}}{\sigma_{i}}+\frac{\tau_{j}}{\sigma_{j}}\right)\right]+\left[\left(\sigma_{i}^{-1}-\sigma_{j}^{-1}\right)+2\left(\frac{\tau_{i}}{\sigma_{i}}-\frac{\tau_{j}}{\sigma_{j}}\right)\right] a_{i j}}{\left[\left(\sigma_{i}^{-1}+\sigma_{j}^{-1}\right)+\left(\sigma_{i}^{-1}-\sigma_{j}^{-1}\right) a_{i j}\right]^{3}},-1 \leq a_{i j} \leq 1$. 